Cubero-Ibáñez, J.; Ibarra-Sáiz, M.S. y Rodríguez-Gómez, G. (2018). Propuesta metodológica de evaluación para evaluar competencias a través de tareas complejas en entornos virtuales de aprendizaje. Revista de Investigación Educativa, 36(1), 159-184.

DOI: http://dx.doi.org/10.6018/rie.36.1.278301

\title{
Propuesta metodológica de evaluación para evaluar competencias a través de tareas complejas en entornos virtuales de aprendizaje
}

\section{Proposal for an assessment methodology to assess skills by using complex tasks within virtual learning environments}

\author{
Jaione Cubero-lbáñez, María Soledad Ibarra-Sáiz y Gregorio Rodríguez-Gómez \\ Grupo EVALfor - Universidad de Cádiz
}

\begin{abstract}
Resumen
Se presenta en este artículo una propuesta metodológica de evaluación del nivel competencial de los estudiantes universitarios en cinco competencias: juicio analítico y crítico, resolución de problemas, sentido ético, toma de decisiones y trabajo en equipo. Se centra la introducción en los dos focos conceptuales en los que se sustenta el estudio, la evaluación de competencias a través de tareas complejas y en un contexto digital con e-rúbricas. En el segundo apartado se contextualiza la propuesta en el Proyecto DevalSimWeb en el que surge, se definen las competencias y se ofrece una descripción de los aspectos fundamentales del Programa Formativo APREVAL-DevalSimWeb. En método se especifican los interrogantes que guian la propuesta, el procedimiento y el diseño metodológico, de acuerdo al método de diseño de experto, de la propuesta de evaluación. Los resultados recogen la propuesta metodológica realizada sobre la base de las tareas complejas y el uso de e-rúbricas que se utilizarán antes y después de la realización
\end{abstract}

Correspondencia: Jaione Cubero Ibáñez, jaione.cubero@uca.es, Universidad de Cádiz, Avda. República Árabe Saharaui s/n, 11519. Puerto Real, Cádiz.

Resultados de la investigación: Comisión Europea Proyecto DevalSimWeb № de contrato DCIALA/19.09.01/11/21526/264-773/ALFAIII(2011)-10. 
del programa formativo para evaluar el nivel competencial de los estudiantes de primer año de carrera. Finaliza el artículo con unas conclusiones y líneas de investigación asociadas a la propuesta. En un anexo se ofrece para cada una de las competencias la descripción de las tareas complejas especificando, antes y después de la implementación del programa, las situaciones/ problema planteadas, los recursos utilizados y las actividades concretas de evaluación junto con las cinco e-rúbricas completas correspondientes a cada competencia.

Palabras clave: evaluación competencial; tareas complejas; e-rúbricas; educación superior.

\section{Abstract}

This article presents a methodology to assess the skill level of university students in five skills: analytical and critical thought, problem solving, sense of Ethics, decision making and teamwork. The introduction focuses on two conceptuals foci in which the study is based, skills assessment through complex tasks and a digital context with e-rubrics. In the second section the proposal is contextualized in the DevalsimWeb Project in which it arises, skills are defined and a description of the fundamental aspects of APREVAL-DevalSimWeb Training Course is offered. In method are specified the questions that guide the proposal, the procedure and the methodological design of the evaluation proposal, according to Expert Desing Method. The results reflect the methodological proposal made on the basis of complex tasks and applying e-rubrics before and after the completion of the training course to assess the skill level of first year university students. The article ends with conclusions and research associated with the proposal. An annex is provided for the description of complex tasks specifying the situations/ problems raised, the resources used and the specific evaluation activities for each skill, before and after implementation of the training course together with the five e-rubrics for each skill.

Keywords: skill evaluation; complex task; e-rubrics; higher education.

\section{Introducción}

La Educación Superior ha ido integrando a lo largo de los últimos años una formación basada en competencias como respuesta a la necesidad de formar estudiantes autorregulados (Dresel, 2015) capaces de transferir sus conocimientos y habilidades a diferentes contextos caracterizados por un entorno dinámico, cambiante y poder adaptarse exitosamente a las exigencias del mercado laboral actual (Peris-Ortiz \& Merigó Lindahl, 2015). Este nuevo paradigma educativo, centrado en los resultados de aprendizaje del estudiante, ha posibilitado nuevos planteamientos relacionados con el diseño, desarrollo y la evaluación de competencias (Tejada \& Ruiz, 2016).

Diferentes autores (Baartman \& Ruijs, 2011; Gómez-Ruiz, Rodríguez-Gómez \& Ibarra-Sáiz, 2013; Kamphorst, Hofman, Jansen \& Terlouw, 2013; Rodríguez-Gómez, Ibarra-Sáiz \& Cubero-Ibáñez, 2018) han evaluado el nivel competencial de los estudiantes a través de la competencia percibida, valiéndose de instrumentos como cuestionarios o autoinformes competenciales que recogen la respuesta de los mismos. Esta medida indirecta de evaluación ha cobrado importancia por la capacidad de los estudiantes para hacer una valoración acertada de su propio nivel competencial (Baartman y Ruijs, 2011) y promover la autorregulación del aprendizaje a través de la reflexión sobre las debilidades y fortalezas de sus propias capacidades (Gómez-Ruiz 
et al., 2013). Sin embargo, Zlatkin-Troitschanskaia, Shavelson \& Kuhn (2015) ponen de manifiesto la necesidad de diseñar e investigar nuevas propuestas de evaluación que permitan evaluar competencias de forma directa, fiable, y válida, ya que se evidencia la falta de métodos basados en demostraciones. Además afirman que existe una falta de métodos innovadores en evaluación, predominando el uso de instrumentos como cuestionarios o pruebas de opción múltiple basadas en lápiz y papel. Es importante por lo tanto, realizar nuevas propuestas e investigaciones que se centren en cómo abordar procesos de evaluación desde posiciones innovadoras (Rodríguez-Gómez \& Ibarra-Sáiz, 2011), diseñando métodos de evaluación competencial basados en las construcciones de los estudiantes e integrando las potencialidades de la tecnología como herramienta para su desarrollo (Tejada \& Ruiz, 2016).

En este trabajo se presenta una propuesta metodológica de evaluación para valorar directamente el nivel competencial, previo y posterior, de estudiantes universitarios de primer año participantes en programas formativos. En ella se presenta a los estudiantes una serie de tareas complejas para su resolución, de forma que se enfrenta al estudiante a situaciones/problemas conectados con la realidad, que le exigen poner en práctica los distintos elementos de la competencia que se desea evaluar.

\section{Evaluación de competencias a través de tareas complejas}

Sobre las tareas de evaluación son varios los autores que han incidido en su importancia para desarrollar una evaluación auténtica (Ashford-Rowe, Herrington \& Brown, 2014; Gulikers, Bastiaens \& Kirschner, 2004). En esta línea RodríguezGómez \& Ibarra-Sáiz (2015), cuando se refieren a los tres retos a los que se enfrenta el enfoque de evaluación como aprendizaje y empoderamiento, indican como uno de ellos las tareas de calidad a través de las cuales se deben desarrollar los otros dos retos, la participación de los estudiantes y la proalimentación. Estas tareas de calidad se basan en los principios de reto, reflexión y transversalidad y se caracterizan por estar contextualizadas en situaciones reales, ser retadoras y transversales; exigir demostraciones y productos de los estudiantes que impliquen tanto un rigor intelectual y pensamiento crítico como la puesta en práctica de diferentes competencias y estrategias. Es decir que propicien en los estudiantes la autorregulación y empoderamiento de su aprendizaje.

La complejidad en la evaluación competencial es comprensible teniendo en cuenta que se evalúa un constructo que integra distintos elementos. Siguiendo a RodríguezGómez \& Ibarra-Sáiz (2011), se concibe el término competencia como un "atributo latente, conocimiento, actitud, habilidad, destreza, y facultad para el desarrollo de una profesión, puesto de trabajo o actuación académica, ejecutando adecuada y correctamente las actuaciones y actividades laborales o académicas exigidas" (p. 70). En la misma línea, Villa y Poblete (2011) lo definen como un conjunto de "conocimientos, técnicas, actitudes, procedimientos, valores que una persona pone en juego en una situación problemática concreta, demostrando que es capaz de resolverla" (p. 148), por ello defendemos un sistema de evaluación que parta de la actuación activa del estudiante, permitiéndole utilizar sus conocimientos de manera creativa para resolver problemas reales (Villardón, 2006). 
Apoyando esta idea, diferentes autores (Fernández March, 2011; Poblete \& Villa, 2011; Ibarra-Sáiz \& Rodríguez Gómez, 2014a; Zabala \& Arnau, 2008) proponen enfrentar a los estudiantes ante problemas que sean reflejo de las situaciones reales en las que se pretende que sean competentes, exigiendo al estudiante movilizar un conjunto de recursos de diferente orden para poder resolverlo. De ahí que en esta propuesta de evaluación se plantean tareas complejas como situación para que, sobre la base de las demostraciones de los estudiantes, se pueda evaluar su nivel competencial.

Ahora bien, evaluar el nivel de adquisición de una competencia no es tarea sencilla, pues requiere la determinación de niveles de dominio y el establecimiento de indicadores y evidencias que permitan ser valoradas (Villa \& Poblete, 2007). Por ello, aunque hasta ahora no se le ha prestado la suficiente atención en los diseños de evaluación competencial (Lester, 2014), es necesario establecer una definición de cada nivel para diferenciar el umbral, de esta forma "se vincula de modo más directo el aprendizaje y/o adquisición/desarrollo de la competencia con su evaluación" (Villa \& Poblete, 2011, p. 152).

En cuanto a los instrumentos adecuados para evaluar competencias a través de tareas complejas se presta especial atención a las rúbricas, ya que permiten valorar aspectos complejos, imprecisos y subjetivos, aportando una evaluación fácilmente interpretable (Buján, Rekalde \& Aramendi, 2011; García, 2014), además de posibilitar la "evaluación del grado del cumplimiento de un atributo ofreciendo la descripción de requisitos para situarse en cada nivel" (Rodríguez-Gómez \& Ibarra-Sáiz, 2011, p.108), aspecto imprescindible para determinar el grado de desarrollo o nivel competencial que presentan los estudiantes en una determinada competencia.

\section{Evaluación con rúbricas en un entorno digital (e-rúbricas)}

El uso de las rúbricas en el contexto de la educación superior es cada vez mayor, como lo ponen de manifiesto las aportaciones de Andreu-Andrés y García-Casas (2014), Hack (2015), Jonnson y Svingby (2007), o Menéndez-Varela y Gregori-Giralt (2016). En la actualidad estamos en un escenario educativo condicionado por la virtualización y digitalización, en el que los entornos virtuales de aprendizaje constituyen el principal soporte electrónico para la formación y el desarrollo en la educación superior (Mueller \& Strohmeier, 2011), pero es preciso poner de manifiesto la necesidad de que la evaluación se adapte al entorno digital coherentemente con los enfoques pedagógicos del siglo XXI, con el fin de desarrollar estudiantes reflexivos y autorregulados (Eyal, 2012). En consecuencia, no basta con la digitalización de antiguas formas o tareas de evaluación (Bennett, 2002), sino que es preciso un cambio tanto en el sistema, como en el planteamiento de las distintas actividades de evaluación (Delgado, 2005).

La incorporación de tecnologías a la evaluación aporta numerosos beneficios. Así, Redecker y Johannessen (2013) resaltan las posibilidades que ofrecen las tecnologías de la información y la comunicación para presentar nuevas tareas y formatos de evaluación más holísticos que permitan evaluar competencias transversales difícilmente 
evaluables con los métodos tradicionales. Sliney y Murphy (2011), por su parte, destacan la potencialidad de simular tareas y escenarios conectados con la realidad donde los estudiantes interactúan y se enfrentan a situaciones cotidianas en contextos virtuales y Bridgeman (2009) destaca la facilidad en la gestión de la información y la rapidez de cálculo.

En el caso del uso de las rúbricas como instrumentos de evaluación, Reddy y Andrade (2010) manifiestan que a veces se conceptualizan como rúbricas algunos instrumentos que no pasan de ser meras escalas de estimación o listas de control. Para considerar un instrumento de evaluación como rúbrica analítica estos autores consideran tres elementos característicos. En primer lugar, que se expliciten los criterios de evaluación, centrados en los resultados de aprendizaje o competencias que serán objeto de evaluación; en segundo lugar, que se presente la calidad de cada criterio mediante la incorporación de descripciones cualitativas de lo que se espera para un determinado nivel o calificación; y por último, que se contemple el sistema de puntuación, a través del establecimiento de rangos o grados para cada una de las descripciones de calidad. Logicamente, las condiciones y requisitos de las rúbricas analíticas, deben mantenerse también cuando se hace uso de las e-rúbricas, es decir, rúbricas utilizadas en un contexto digital de forma que se facilite la gestión y la rapidez pero, también, se mantenga la coherencia con el enfoque evaluativo en el que se sustentan.

\section{Contexto del estudio: El proyecto DevalSimWeb}

Este estudio se enmarca en un escenario internacional contextualizado en el Proyecto DevalSimWeb-Desarrollo de competencias profesionales a través de la evaluación participativa y la simulación utilizando herramientas web, en el que han participado dos socios europeos (Universidad de Cádiz y Durham University) y cuatro de América Latina (Pontificia Universidad Católica de Ecuador-Sede Ibarra, Universidad de Antioquia [Colombia], Universidad de Costa Rica y Universidad Nacional Agraria [Nicaragua]). A través de este proyecto se pretendía mejorar la calidad de la Educación Superior en América Latina (AL) mediante el desarrollo de la competencia evaluadora del profesorado y de los estudiantes en un conjunto de competencias transversales, consideradas necesarias para el éxito académico y la integración laboral de los estudiantes en un entorno caracterizado por el uso de las TIC mediante redes sociales y de conocimiento.

Con la intención de innovar y propiciar cambios en los modelos evaluativos de las instituciones universitarias de América Latina, una de las acciones se centró en diseñar e implementar varios programas formativos, dirigidos tanto al profesorado (1 programa formativo) (Ibarra-Sáiz \& Rodríguez-Gómez, 2014b) como al estudiantado ( 2 programas formativos), incorporando premisas de enfoques innovadores en evaluación y diseñados e implementados en un contexto de aprendizaje mixto donde predomina el componente virtual utilizando diferentes recursos y herramientas tecnológicas. 


\section{Catálogo de competencias transversales a la evaluación}

Como punto de partida para el diseño de los tres programas formativos, se consensuaron y definieron las competencias que iban a ser objeto de los programas formativos y se elaboró el Catálogo de Competencias Transversales a la EvaluaciónDevalSimweb (Ibarra-Sáiz, Rodríguez-Gómez \& García-Jiménez, 2013), resultado de diversos estudios previos en los países socios de América Latina, en el cual se definen las cinco competencias seleccionadas como transversales a la evaluación (Tabla 1), y se establecen tres niveles de desarrollo (Tabla 2). Representadas mediante una matriz, se especifican también las actuaciones asociadas a cada una de las competencias, clasificándolas de acuerdo a sus facetas (dimensiones que componen la competencia) y en progresión lógica en el nivel competencial correspondiente. En esta especificación se siguió el manual The Student Evaluation Standards (Joint Committee on Standards for Educational Evaluation [JCSEE], 2002) y se realizó una validación de contenido en la que participaron un total de 43 jueces expertos internacionales.

Tabla 1

Definición de las competencias transversales a la evaluación (Ibarra-Sáiz et al., 2013)

Competencias

Juicio analítico y crítico

Resolución de problemas

Sentido ético

Toma de decisiones

Trabajo en equipo

\section{Definición}

Actúa sistemáticamente, establece jerarquías y utiliza herramientas para el análisis; interroga las situaciones que se le presentan y argumenta la pertinencia de los juicios para tomar decisiones en el marco de su desempeño.

Identifica, analiza, construye criterios, aplica conocimientos ante situaciones específicas, toma decisiones y valora posibles riesgos.

Actúa responsablemente de acuerdo a principios axiológicos universales y respetando contextos culturales diversos.

Actúa oportuna, eficaz y eficientemente, ante diversas situaciones de forma ágil y proactiva, apoyándose en información relevante que facilite la identificación, comprensión, evaluación y asunción de ciertos riesgos en situaciones de incertidumbre, eligiendo la mejor alternativa, mediante la consulta a las fuentes más adecuadas, contrastándolas e integrándolas.

Se compromete responsablemente y colabora con otras personas en el logro de objetivos comunes, compartiendo recursos y conocimientos, armonizando diversos intereses, valorando las oportunidades y contribuyendo así a un clima de trabajo que facilite la ejecución y desarrollo de tareas. 
Tabla 2

Niveles competenciales de acuerdo al Catálogo de Competencias Transversales a la Evaluación-DevalsimWeb (Ibarra-Sáiz, et al., 2013)

\begin{tabular}{ll}
\hline $\begin{array}{l}\text { Nivel } \\
\text { competencial }\end{array}$ & \multicolumn{1}{c}{ Descripción } \\
\hline Cotidiano & $\begin{array}{l}\text { Indica un conocimiento intuitivo y dominio básico de la evaluación que } \\
\text { permite desarrollar actividades elementales y comprensivas sobre las ac- } \\
\text { ciones evaluativas a desarrollar. }\end{array}$ \\
$\begin{array}{l}\text { Reflexivo } \\
\text { (técnico) }\end{array}$ & $\begin{array}{l}\text { Se establecen análisis y conexiones entre las acciones, utilizando proce- } \\
\text { dimientos, estrategias y recursos que permiten explicaciones sensatas y } \\
\text { fundamentadas sobre los resultados obtenidos. }\end{array}$ \\
$\begin{array}{l}\text { Comprensivo } \\
\text { (integrador y } \\
\text { creativo) }\end{array}$ & $\begin{array}{l}\text { Existe integración, aplicación, creatividad y criticidad en las decisiones } \\
\text { tomadas respecto a las acciones y resultados de la evaluación, argumen- } \\
\text { tando las mismas y facilitando una información objetiva, fiable, útil y } \\
\text { orientada a la mejora. }\end{array}$ \\
\hline
\end{tabular}

\section{Programa Formativo APREVAL-DevalSimWeb: Aprender Evaluando en la Educa- ción Superior}

El Programa Formativo APREVAL-DevalSimWeb: Aprender evaluando en la Educación Superior (Rubio, Tocaín, \& Ruiz, 2014), de carácter semipresencial, se centra en la capacitación de los estudiantes para abordar procesos de e-evaluación a través de herramientas electrónicas y simulación, con el fin de incrementar su nivel competencial y favorecer así su éxito académico.

Los destinatarios, estudiantes universitarios de primer año pertenecientes a las universidades socias de AL participantes en el Proyecto DevalSimWeb, se caracterizan por realizar la formación de manera voluntaria, principalmente motivados por aprender y sin mayores requisitos que disponer de acceso a internet y conocimientos de manejo básico en plataformas virtuales.

El marco lógico del programa se centra en cuatro aspectos fundamentales que guían el proceso, representados en la Figura 1.

El programa tiene como objeto desarrollar y trabajar las cinco competencias consideradas transversales a la evaluación recogidas en el Catálogo de Competencias Transversales a la Evaluación-DevalSimWeb. Tanto las competencias seleccionadas como los contenidos/aspectos referentes a la evaluación (principios de la evaluación, criterios de evaluación, instrumentos de evaluación y modalidades de evaluación) se desarrollan y se ponen en práctica explícitamente a través de diferentes tareas de calidad.

Los estudiantes participan en el programa formativo en procesos de e-evaluación a través del uso del servicio web EvalCOMIX ${ }^{\circledR}$ (http://evalcomix.uca.es/) y otras herramientas electrónicas como foros, wikis y juegos de simulación. 


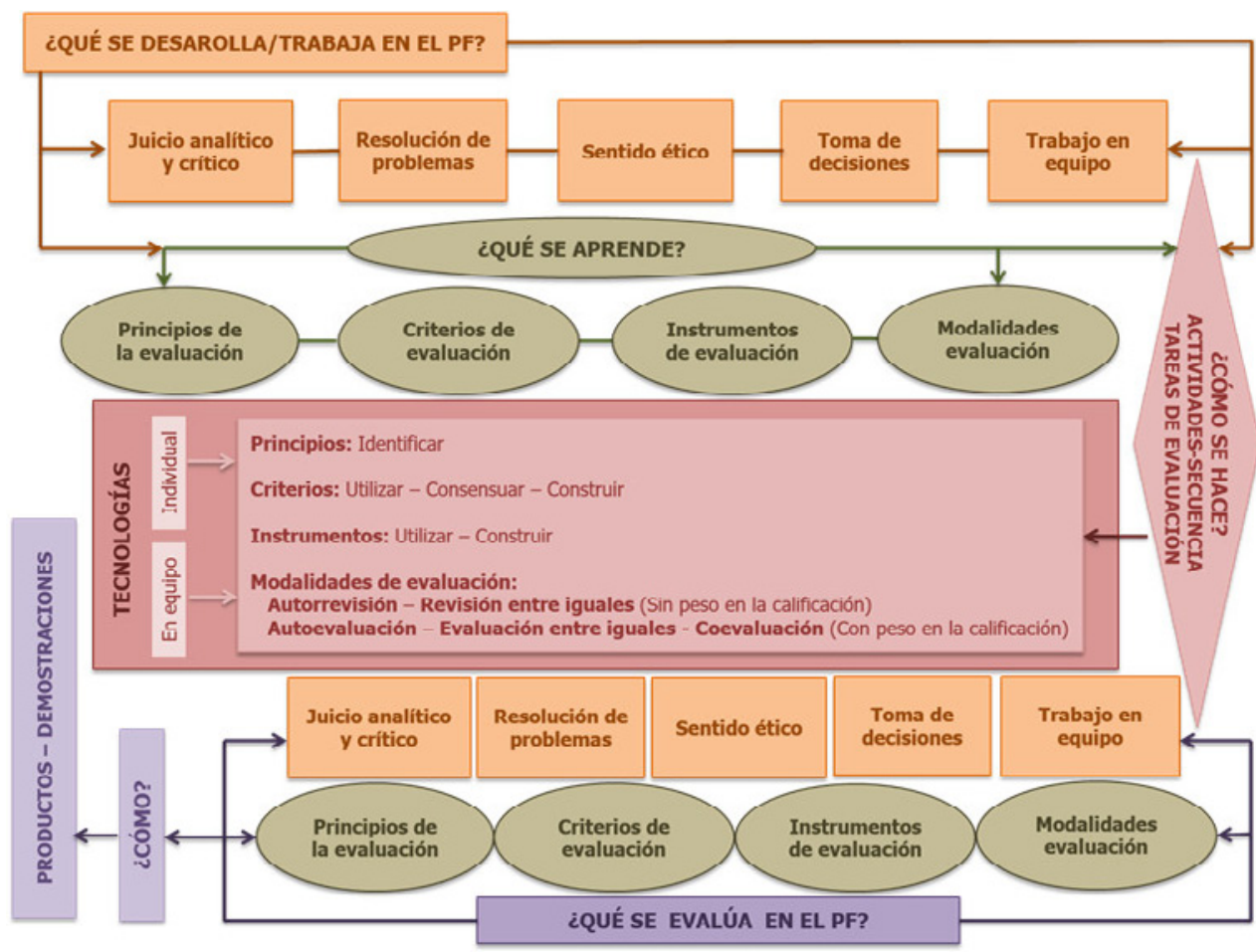

Figura 1. Marco lógico del PF APREVAL-DevalSimWeb

(Ibarra-Sáiz \& Rodríguez- Gómez, 2014a, p. 18).

\section{Método}

\section{Objetivos}

El interés del estudio reside en diseñar una propuesta metodológica para evaluar el nivel competencial de los estudiantes de primer año universitario participantes en el PF APREVAL-DevalSimWeb, basada en la construcción de respuestas directas de los estudiantes en un contexto virtual.

Específicamente se pretendía dar respuesta a los siguientes interrogantes:

- ¿Qué plataforma podemos utilizar como entorno virtual de evaluación?

- ¿Qué tareas se pueden diseñar para evaluar el nivel competencial de los estudiantes en las cinco competencias consideradas transversales a la evaluación (antes y después de participar en el programa formativo) a través de entornos virtuales?

- ¿Qué instrumentos se pueden utilizar para medir el nivel competencial de los estudiantes?

- ¿Cómo analizar el nivel competencial de los estudiantes? 


\section{Procedimiento}

A continuación se presenta la descripción del proceso seguido para diseñar la propuesta metodológica de evaluación competencial realizada a partir del Método de Diseño de Experto (Álvarez-Rojo, 1998). En la Figura 2 se describen las fases seguidas para realizar la propuesta que se describirá posteriormente.

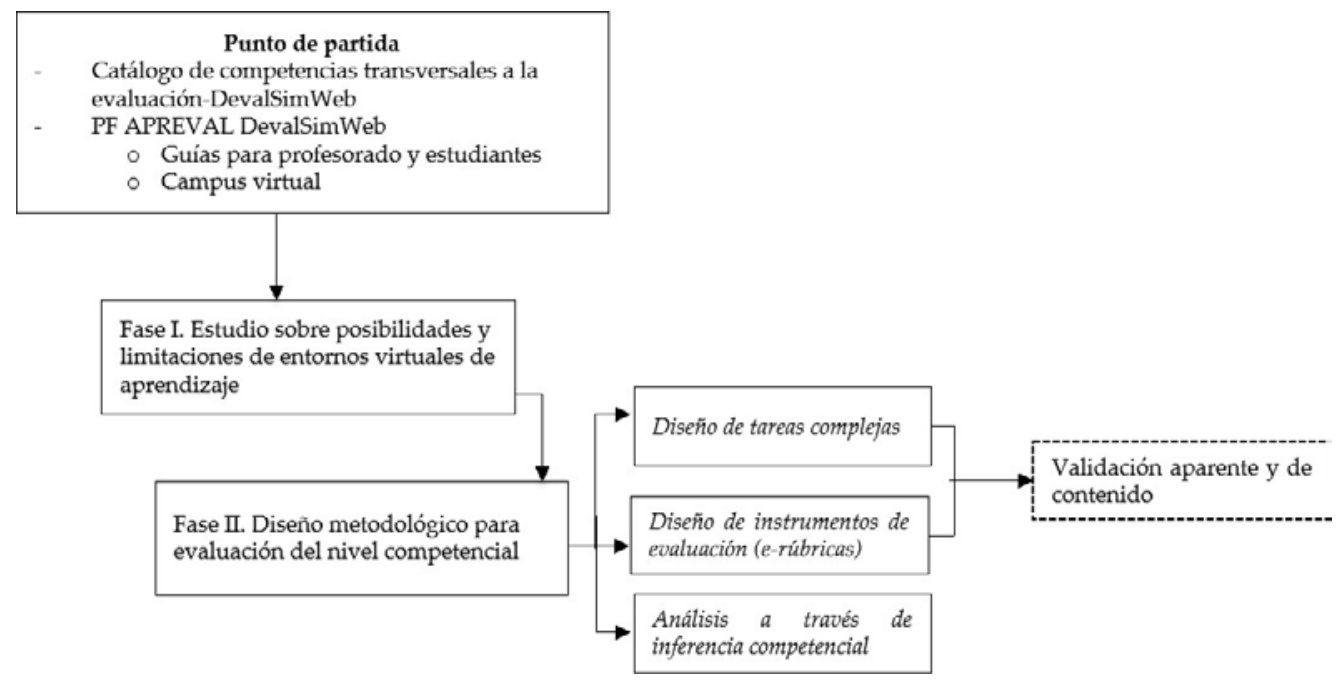

Figura 2. Fases para la elaboración del diseño metodológico de evaluación competencial del PF APREVAL-DevalSimWeb.

\section{Proceso de validación}

Entre los diferentes métodos comúnmente utilizados para la validación mediante jueces expertos (Corral, 2009; Litwin, 2003) como el método de agregados individuales, el método Delphi o la técnica de grupo nominal, en esta investigación se ha optado por el método de consenso grupal, ya que mediante el mismo se evitan los sistemas de votación. Este método permite que a través de un desarrollo iterativo e incremental en sucesivos ciclos se consiga el objetivo de llegar a un producto final con el que están de acuerdo todos los expertos consultados.

En este estudio el método de consenso grupal mediante jueces expertos se ha utilizado, en primer lugar, para la validación aparente y de contenido de las 10 tareas complejas ( 5 de pretest y 5 de postest referidas a cada una de las 5 competencias seleccionadas). Así mismo, se ha utilizado para la validación de los 5 instrumentos (e-rúbricas) diseñados para la evaluación de cada una de las competencias. Las secuencias de diseño de las tareas complejas y de las e-rúbricas se presentan posteriormente, y su propuesta consensuada final se incluyen en el anexo.

En cada uno de estos 2 procesos de validación han participado 9 expertos pertenecientes a 6 universidades de 5 países diferentes. Estos expertos fueron propuestos 
por los diferentes equipos de investigación participantes en el proyecto, tomando en consideración los criterios propuestos por Skjong y Wenthworth (2000) de experiencia en la realización de juicios y toma de decisiones, disponibilidad y motivación para participar en el proceso e imparcialidad. Sobre la base de una propuesta inicial se sucedieron diversos ciclos iterativos cualitativos e incrementales inter-jueces hasta llegar al consenso final.

\section{Fase I: Estudio sobre posibilidades y limitaciones de entornos virtuales de aprendizaje}

El carácter virtual del programa formativo, y la naturaleza de los resultados a medir (competencias), planteó el reto de diseñar un procedimiento de evaluación competencial a través de un entorno virtual de aprendizaje. La condición previa fundamental para obtener ventajas mediante el uso de un entorno virtual de aprendizaje, es la correcta selección del sistema adecuado a los requerimientos (Mueller \& Strohmeier, 2011). Por ello, en este caso, además de buscar una plataforma de código abierto, se basó en las cuatro características básicas e imprescindibles que define Boneu (2007) para seleccionar adecuadamente una plataforma virtual: interactividad, flexibilidad, escalabilidad y estandarización. Igualmente otros aspectos como la calidad técnica, organizativa y creativa, comunicacional y didáctica (Belloch, 2012) fueron relevantes en la selección. Finalmente tras revisar las diferentes plataformas existentes y valorar las posibilidades y limitaciones de cada una teniendo en cuenta los criterios citados, se optó por la plataforma Moodle, creando el espacio virtual "Nivel Competencial APREVAL", e incorporando la integración del servicio web EvalCOMIX ${ }^{\circledR}$.

\section{Fase II: Diseño metodológico de evaluación competencial}

Para diseñar las tareas de evaluación, se tomó como referencia el modelo de evaluación basado en situaciones complejas (Figura 3) propuesto por Poblete y Villa (2011).

En consonancia, se decide diseñar una tarea compleja diferente por competencia y momento de evaluación, es decir un total de diez tareas complejas (5 para la evaluación previa y otras 5 para la evaluación posterior).

Seguidamente se especifican los pasos que guiaron el diseño de estas tareas complejas (Figura 4):

1. Especificar las condiciones que debe reunir una tarea para que sea compleja. Se basaron en las condiciones propuestas por Fernández March (2011):

- Análisis de diferentes dimensiones

- Aplicación a más de una situación para facilitar la transferencia

- Cercanas a la realidad

- Movilización de recursos diversos internos (saber, saber hacer y saber ser) y externos (solicitados de manera autónoma por el estudiante)

- Utilización de juicio y discernimiento rigurosos.

- Indicadores de desarrollo (niveles de dominio)

- Intervención en el proceso de evaluación de diferentes agentes (Concordancia) 


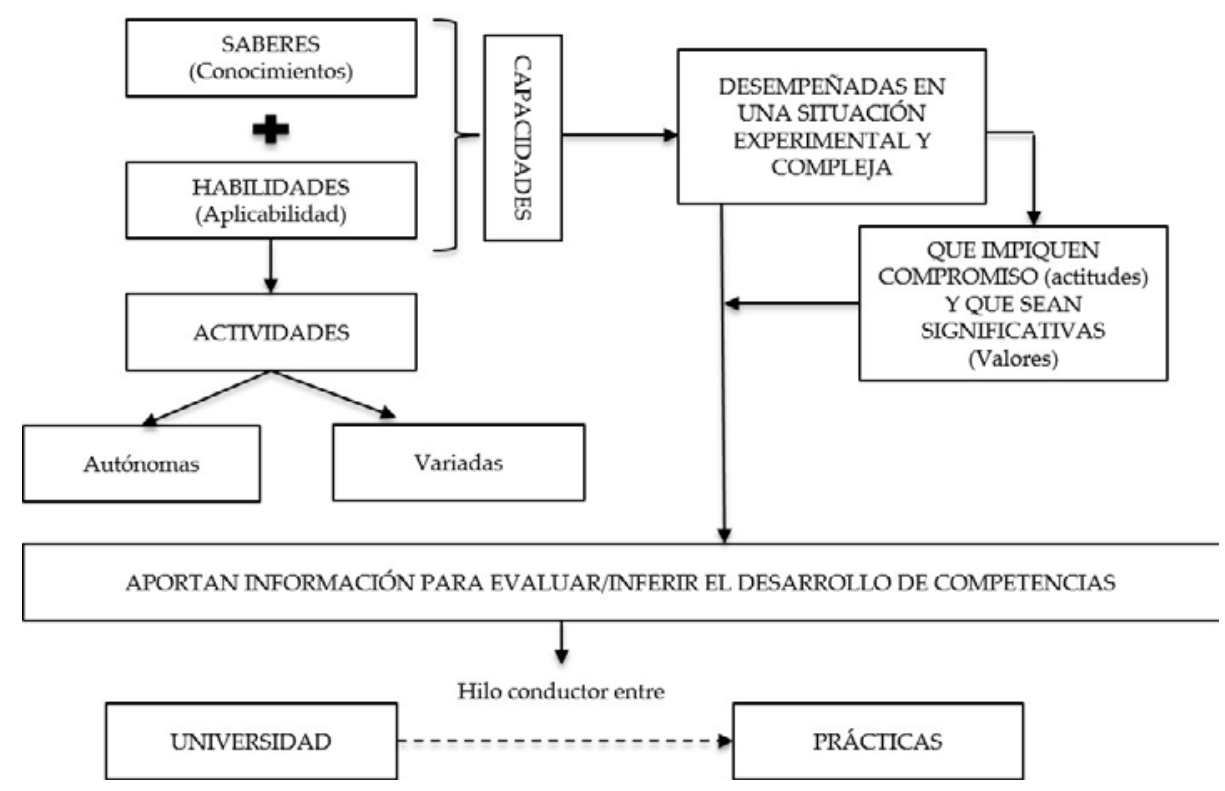

Figura 3. Modelo de evaluación basado en situaciones complejas a partir de Poblete y Villa (2011, p. 17).

2. Determinar la tipología de las tareas de evaluación en función de las competencias a evaluar. Para ello se siguió la clasificación de actividades propuesta por Delgado y Oliver (2006).

3. Seleccionar/diseñar las situaciones/problema a resolver para cada una de las competencias. Para determinar el contenido y las preguntas de evaluación, se tuvieron en cuenta las definiciones, niveles de desarrollo y actuaciones descritas en el Catálogo de Competencias Transversales a la Evaluación-DevalSimWeb.

4. Buscar y seleccionar diferentes recursos mediante los cuales puedan ser presentadas. Siguiendo a Zabala y Arnau (2008) éstas pueden ser representadas mediante acontecimientos, textos periodísticos o científicos, sucesos, conflictos. En esta propuesta la naturaleza de las tareas varía dependiendo del caso concreto de cada competencia, por lo que fue necesario buscar y seleccionar diferentes recursos para poder presentar las situaciones/problemas.

5. Revisión general de aspectos formales. Adecuación del lenguaje y estimación del tiempo de resolución de cada tarea.
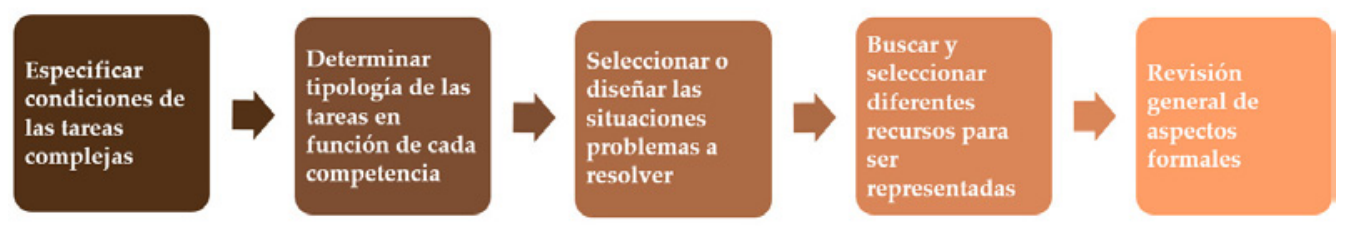

Figura 4. Secuencia para el diseño de las tareas complejas. 
Como instrumento para evaluar el nivel competencial de los estudiantes se optó por el uso de e-rúbricas, las cuales permiten especificar de manera anticipada las dimensiones que integra una tarea así como los niveles de desempeño a alcanzar (De la Cruz \& Abreu, 2014). Tomando como ejemplo la propuesta de evaluación de competencias genéricas de Villa y Poblete (2007) y teniendo en cuenta los niveles, facetas y actuaciones establecidas en el Catálogo de Competencias Transversales a la EvaluaciónDevalSimWeb, se diseñaron un total de cinco e-rúbricas utilizando para ello el servicio web EvalCOMIX ${ }^{\circledR}$. Es decir, se empleó el mismo instrumento de evaluación para cada una de las competencias en el pretest y postest.

Tanto el diseño de tareas complejas, diez, como el diseño de los instrumentos de evaluación, cinco, fueron objeto de validación aparente y de contenido mediante acuerdo interjueces. Ambas validaciones se realizaron a través de la herramienta de trabajo colaborativo BSCW, donde se subieron los materiales elaborados, y se establecieron dos rondas de revisión por jueces.

\section{Resultados}

Los resultados de este estudio no se refieren al nivel competencial alcanzado por los estudiantes, sino a la presentación de una propuesta metodológica para evaluar el desarrollo competencial de estudiantes universitarios de primer año. Específicamente, la propuesta se basa en tres aspectos principales:

- disponer de un entorno virtual de evaluación adecuado,

- diseño y uso de tareas complejas y

- elaboración y uso de e-rúbricas analíticas.

Como se ha destacado anteriormente, el entorno virtual de evaluación que se propone se construye sobre un entorno Moodle, mejorado con la integración del servicio web EvalCOMIX ${ }^{\circledR}$ a través de la API EvalCOMIX_MD® (Figura 5). Esta arquitectura permite el uso de instrumentos de evaluación y diferentes modalidades evaluativas de manera fácil e integrada.

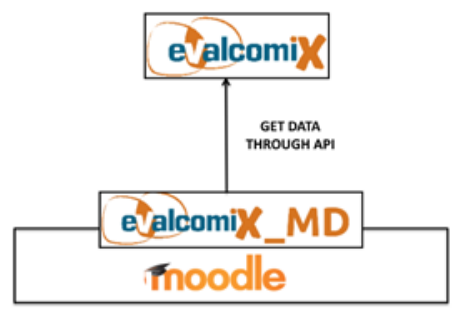

Figura 5. Arquitectura de integración del servicio web EvalCOMIX ${ }^{\circledR}$.

La función de las tareas complejas y la e-rúbricas en esta propuesta metodológica de evaluación se ilustra en la Figura 6 y, como podemos observar, se centra en evaluar el nivel competencial de los estudiantes antes (evaluación nivel competencial inicial) y 
después de participar en un programa formativo (evaluación nivel competencial final). En este caso, esta evaluación se realiza sobre las cinco competencias seleccionadas como transversales a la evaluación (juicio analítico y crítico, resolución de problemas, sentido ético, toma de decisiones y trabajo en equipo).

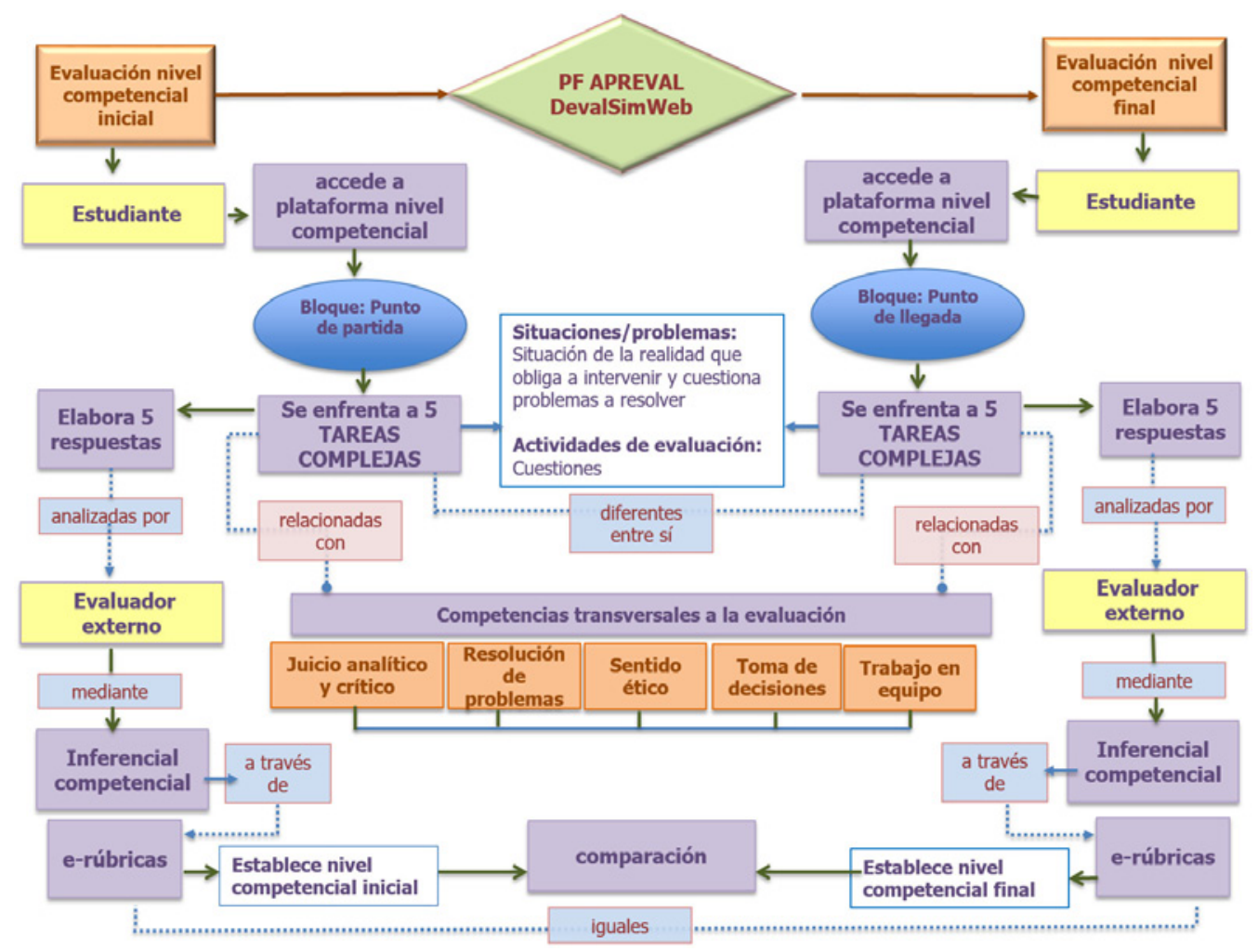

Figura 6. Propuesta metodológica de evaluación competencial para el PF APREVAL-DevalSimWeb.

La actividad de los estudiantes y evaluadores, se desarrolla en el espacio virtual de evaluación, al que se le ha denominado "Nivel Competencial APREVAL", que se divide en dos bloques (pestañas), según el momento de la ejecución de las tareas complejas y su correspondiente evaluación: Punto de partida, en el que se alojan las tareas complejas iniciales y sus actividades; y Punto de llegada, después de la finalización del programa formativo, donde se presentan las tareas complejas finales y sus actividades concretas (Figura 7).

En ambos casos, los estudiantes deben acceder a la plataforma y enfrentarse a cinco tareas complejas (una por cada competencia) las cuales plantean una situación problema y unas cuestiones relacionadas a las que el estudiante debe responder (Figura 8). Esas respuestas son registradas en la plataforma para ser posteriormente evaluadas por un evaluador externo. 


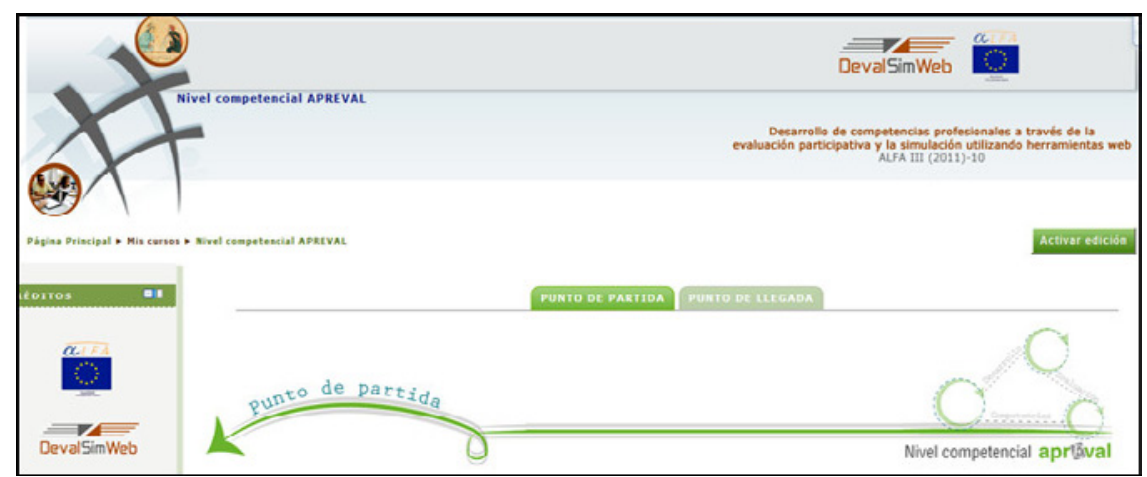

Figura 7. Captura de los bloques "Punto de partida" y "Punto de llegada" del Campus Virtual "Nivel competencial APREVAL".

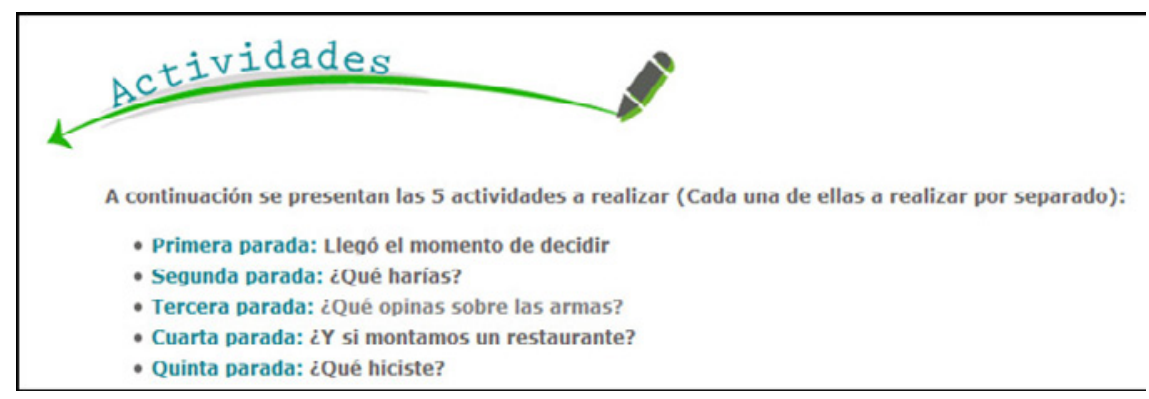

Figura 8. Captura de las cinco tareas correspondientes al bloque "Punto de partida".

Como se puede apreciar en la Figura 9, cada tarea presenta tres elementos imprescindibles para facilitar la comprensión de los estudiantes: una pequeña introducción, el recurso donde se presenta la tarea compleja (visual o escrito) y el espacio de entrega de producciones (formato pdf), donde se especifican las actividades y cuestiones a responder.

\section{Segunda parada: ¿QUE HARIAS?}

Lee el documento titulado Texto Segunda parada: ¿QUÉ HARÍAS?

Archivo Texto Segunda parada ¿QUÉ HARÍAS?

¿Qué harías?

Figura 9. Captura de la presentación de una de las tareas complejas. 
En la Tabla 3 se presenta un resumen de las tareas complejas diseñadas atendiendo a sus cinco elementos principales: competencias, tipologías, actividades y recursos, siendo los últimos tres diferentes en el pretest y postest. Con el objeto de ofrecer una información detallada se presenta, en el anexo, para cada una de las cinco competencias del estudio (Juicio analítico y crítico, Resolución de problemas, Sentido ético, Toma de decisiones y Trabajo en equipo) una descripción de las tareas complejas diseñadas, haciendo referencia a la situación planteada a los estudiantes, el recurso utilizado y la propia actividad de evaluación.

Tabla 3

Tipología, actividad y recurso de las tareas complejas diseñadas

\begin{tabular}{|c|c|c|c|c|c|}
\hline \multirow[b]{2}{*}{ Competencias } & \multirow[b]{2}{*}{ Tipología } & \multicolumn{2}{|c|}{ Punto de partida (pretest) } & \multicolumn{2}{|c|}{ Punto de llegada (postest) } \\
\hline & & Actividad & Recurso & Actividad & Recurso \\
\hline $\begin{array}{l}\text { Juicio analítico y } \\
\text { crítico }\end{array}$ & $\begin{array}{l}\text { Comentario } \\
\text { crítico }\end{array}$ & $\begin{array}{c}\text { ¿Qué opinas } \\
\text { sobre las } \\
\text { armas? }\end{array}$ & $\begin{array}{l}\text { Artículo web } \\
\text { de opinión }\end{array}$ & $\begin{array}{c}\text { Ley, ciencia } \\
\text { y conciencia } \\
\text { ante el aborto }\end{array}$ & $\begin{array}{c}\text { Artículo web } \\
\text { de opinión }\end{array}$ \\
\hline $\begin{array}{l}\text { Resolución de } \\
\text { problemas }\end{array}$ & $\begin{array}{l}\text { Resolución } \\
\text { caso }\end{array}$ & ¿Qué hiciste? & Texto escrito & $\begin{array}{l}\text { ¿De quién es } \\
\text { el pececito? }\end{array}$ & Texto escrito \\
\hline Sentido ético & $\begin{array}{l}\text { Dilema } \\
\text { moral }\end{array}$ & ¿Qué harías? & Texto escrito & ¿Qué harías? & Texto escrito \\
\hline $\begin{array}{l}\text { Toma de deci- } \\
\text { siones }\end{array}$ & $\begin{array}{l}\text { Supuesto } \\
\text { práctico }\end{array}$ & $\begin{array}{l}\text { Es tiempo de } \\
\text { decidir }\end{array}$ & $\begin{array}{l}\text { Fragmento } \\
\text { película }\end{array}$ & $\begin{array}{c}\text { Es tiempo de } \\
\text { decidir }\end{array}$ & $\begin{array}{l}\text { Fragmento } \\
\text { película }\end{array}$ \\
\hline $\begin{array}{l}\text { Trabajo en } \\
\text { equipo }\end{array}$ & $\begin{array}{l}\text { Supuesto } \\
\text { práctico }\end{array}$ & $\begin{array}{l}\text { ¿Montamos un } \\
\text { restaurante? }\end{array}$ & $\begin{array}{c}\text { Fragmento } \\
\text { programa TV }\end{array}$ & ¿De turismo? & $\begin{array}{l}\text { Fragmento } \\
\text { programa TV }\end{array}$ \\
\hline
\end{tabular}

El periodo para realizar las tareas de nivel competencial previo (pretest) se establece durante la semana anterior al comienzo del programa formativo, y el nivel competencial posterior (postest) una semana después de la finalización del mismo. De este modo, los estudiantes pueden acceder a la plataforma libremente durante este espacio de tiempo para construir sus respuestas, aportando flexibilidad al proceso.

Otro aspecto esencial de la propuesta metodológica reside en los instrumentos de evaluación que se utilizan para valorar el nivel competencial de los estudiantes en cada una de las competencias. En este caso se emplean cinco e-rúbricas cuya estructura se presenta en la Tabla 4. El diseño con EvalCOMIX ${ }^{\circledR}$ se adjunta en el anexo organizadas junto con la tarea compleja correspondiente a las producciones que se valoran.

Las respuestas de los estudiantes a las tareas complejas, es decir, sus producciones se registran en el sistema y la valoración de las mismas se efectúa mediante el uso de las 
e-rúbricas utilizando para ello el servicio web EvalCOMIX ${ }^{\circledR}$. Mediante el uso integrado de EvalCOMIX ${ }^{\circledR}$ y el libro de calificaciones de Moodle, se permite la comparación de los niveles competenciales incial y final de cada estudiante en cada una de las competencias, pudiéndose evidenciar si hay alguna modificación entre ambos momentos.

Tabla 4

Estructura de las e-rúbricas.

\begin{tabular}{lccc}
\hline \multirow{2}{*}{ Facetas/dimensiones } & \multicolumn{3}{c}{ Niveles competenciales } \\
\cline { 2 - 4 } Cuestionamiento & Cotidiano & Reflexivo & Comprensivo \\
& Actuaciones & Actuaciones & Actuaciones \\
Argumentación & asociadas & asociadas & asociadas \\
& Actuaciones & Actuaciones & Actuaciones \\
Criticismo & asociadas & asociadas & asociadas \\
& Actuaciones & Actuaciones & Actuaciones \\
& asociadas & asociadas & asociadas \\
\hline
\end{tabular}

\section{Discusión y conclusiones}

Este trabajo presenta una propuesta metodológica para evaluar el nivel competencial de los estudiantes en cinco competencias transversales a la evaluación en un contexto virtual de aprendizaje. Mientras hay autores que basan sus investigaciones en las percepciones de los actores (Baartman \& Ruijs, 2011; Gómez-Ruiz et al., 2013; Kamphorst et al., 2013; Rodríguez-Gómez et al., 2018) es importante dar voz a las construcciones de los estudiantes, es decir, a su manera de enfrentarse a los retos de cuestionamientos que les puede ayudar a afrontar soluciones y respuestas a ciertas situaciones reales con las que se encuentran cotidianamente.

Hasta el momento se ha prestado más atención a demostrar los beneficios del uso de la e-evaluación que en construir propuestas metodológicas prácticas para su implementación (Tomas, Borg \& McNeil, 2015), por ello el valor de este estudio consiste en arrojar luz respecto a las tareas, recursos e instrumentos que pueden emplearse para evaluar el nivel competencial de los estudiantes universitarios en un entorno virtual de aprendizaje.

De acuerdo al objeto de este estudio, presentar una propuesta metodológica basada en la evaluación del nivel competencial de estudiantes a través de la realización de tareas complejas de evaluación y evaluando mediante e-rúbricas, se destacan los siguientes aspectos claves innovadores de la propuesta descrita:

- Construcción de un escenario de e-evaluación competencial integrando las tecnologías como herramientas de apoyo útiles para este fin (Olmos Migueláñez \& Rodríguez Conde, 2011). 
- Presentación de tareas de evaluación a través de diferentes componentes multimedia (Liu \& Li, 2014), rompiendo con modelos de presentación lineal y favoreciendo la recreación de la realidad mediante entornos virtuales.

- Enfrentar a los estudiantes a la resolución de tareas complejas, situaciones o problemas conectados con la realidad, exigiendo en los estudiantes un pensamiento analítico y reflexivo de alto nivel que podrán transferir posteriormente a otros contextos (Ibarra-Sáiz, Rodríguez-Gómez \& García-Jiménez, 2016).

- Empleo de rúbricas análiticas en Educación Superior (Hack, 2015) y hacerlo a través de herramientas tecnológicas convirtiéndose en e-rúbricas (Ibarra-Sáiz \& Rodríguez-Gómez, 2017).

Ese trabajo es un acercamiento para poner en práctica una evaluación digital coherente con los enfoques pedagógicos del siglo XXI (Eyal, 2012), no obstante, no se pueden obviar ciertas limitaciones que presenta la propuesta como el tiempo que exige y alto nivel de capacitación por parte del evaluador a la hora de realizar el juicio valorativo para inferir el nivel competencial.

Es preciso considerar que la generalización de una propuesta metodológica como la presentada exige un elevado compromiso institucional ya que, en primer lugar, es necesario disponer de los recursos y herramientas tecnológicos necesarios, integrados en un espacio virtual de aprendizaje de fácil utilización por parte del profesorado y los estudiantes; y en segundo lugar, se hace del todo imprescindible procesos de alfabetización digital (Eyal, 2012) mediante actividades formativas que permitan convencer al profesorado y a los estudiantes de las ventajas de este tipo de estrategias de e-evaluación.

Para optimizar esta propuesta será necesario realizar nuevas investigaciones y desarrollos que faciliten la integración y fácil utilización de los recursos y herramientas tecnológicos en los procesos de evaluación competencial, favoreciendo así el empoderamiento personal, la participación y el desarrollo de competencias transversales de los estudiantes.

\section{Referencias}

Álvarez-Rojo, V. (1998). Diseño de programas de orientación. En X. Salvador \& M.L. Rodicio (Eds.), Actas del Simposium sobre Orientación: ¿Para onde camiña a orientación? (pp. 142-168). A Coruña: Universidade de A Coruña

Andreu-Andrés, M. A., \& García-Casas, M. (2014). Evaluación del pensamiento crítico en el trabajo en grupo. Revista de Investigación Educativa, 32(1), 203-222. doi: http:// dx.doi.org/10.6018/rie.32.1.157631

Ashford-Rowe, K., Herrington, J., \& Brown, C. (2014). Establishing the critical elements that determine authentic assessment. Assessment $\mathcal{E}$ Evaluation in Higher Education, 39(2), 205-222. doi: http://dx.doi.org/10.1080/02602938.2013.819566

Baartman, L., \& Ruijs, L. (2011). Comparing students' perceived and actual competence in higher vocational education. Assessment $\mathcal{E}$ Evaluation in Higher Education, 36(4), 385-398. doi: http://dx.doi.org/10.1080/02602938.2011.553274

Belloch, C. (2012). Entornos virtuales de aprendizaje. Unidad de Tecnología Educativa (UTE). Universidad de Valencia. Recuperado de https://goo.gl/TaUViS 
Bennett, R.E. (2002). Inexorable and inevitable: The continuing story of technology and assessment. Journal of Technology, Learning and Assessment 1(1). Recuperado de https://goo.gl/52v5ja

Boneu, J.M. (2007). Plataformas abiertas de e-learning para el soporte de contenidos educativos abiertos. Revista de Universidad y Sociedad del Conocimiento 4(1). Recuperado de https://goo.gl/SV0Ja

Bridgeman, B. (2009). Experiences from large-scale computer-based testing in the USA, in F. Scheuermann \& J. Björnsson (Eds.), The Transition to Computer-Based Assessment. Luxembourg, Office for Official Publications of the European Communities.

Buján, K., Rekalde, I., \& Aramendi, P. (2011). Las rúbricas de evaluación en formación de competencias. En K. Bujan; I. Rekalde \& P. Aramendi (Coords.) La evaluación de competencias en la educación superior (pp. 75-97). Sevilla: MAD, S.L.

Corral, Y. (2009). Validez y confiabilidad de los instrumentos de investigación para la recolección de datos. Revista de Ciencias de la Educación, 19(33), 228-247. Recuperado de https://es.scribd.com/doc/48628674/Validez-y-confiabilidad-de-instrumentos

De la Cruz, G., \& Abreu, L.F. (2014). Rúbricas y autorregulación: pautas para promover una cultura de la autonomía en la formación profesional terciaria. REDU: Revista de Docencia Universitaria, 12, 31-48. Recuperado de https://dialnet.unirioja.es/ ejemplar/365061

Delgado, A.M. (Dir.). (2005). Competencias y diseño de la evaluación continua y final en el Espacio Europeo de Educación Superior. Programa de Estudios y Análisis, Ministerio de Educación y Ciencia (MEC). Recuperado de https://goo.gl/BZgFzr

Delgado, A.M., \& Oliver, R. (2006). La evaluación continua en un nuevo escenario docente. RUSC. Universities and Knowledge Society Journal, 3(1). Recuperado de https://goo.gl/rv2sCi

Dresel, M.G. (2015). Competencies for successful self-regulated learning in higher education: structural model and indications drawn from expert interviews. Studies in Higher Education, 40(3), 454-470. Recuperado de http://www.tandfonline.com/doi/ abs/10.1080/03075079.2015.1004236

Eyal, L. (2012). Digital Assessment Literacy - the Core Role of the Teacher in a Digital Environment. Educational Technology E Society, 15, 37-49. Recuperado de http://www. ifets.info/journals/15_2/5.pdf

Fernández March, A. (2011). La evaluación orientada al aprendizaje en un modelo de formación por competencias en la enseñanza universitaria. En K. Bujan, I. Rekalde \& P. Aramendi (Coords.), La evaluación de competencias en la educación superior (pp. 37-57). Sevilla: MAD, S.L.

García, M.P. (2014). La evaluación de competencias en Educación Superior mediante rúbricas: un caso práctico. Revista Electrónica Interuniversitaria de Formación del Profesorado 17(1), 87-106. doi: http://dx.doi.org/10.6018/reifop.17.1.198861

Gómez-Ruiz, M.A., Rodríguez-Gómez, G., \& Ibarra-Sáiz, M.S. (2013). Desarrollo de las competencias básicas de los estudiantes de Educación Superior mediante la e-Evaluación orientada al aprendizaje. RELIEVE. Revista Electrónica de Investigación y Evaluación Educativa, 1(19), 1-17. Recuperado de https://goo.gl/JMXbAx 
Gulikers, J., Bastiaens, T., \& Kirschner, P. (2004). A five-dimensional framework for authentic assessment. Educational Technology Research and Development, 52(3), 67-86. Recuperado de https://racetothetopvolusia.wikispaces.com/file/view/A+Five+Dime nsional+Framework+for+Authentic+Assessment.pdf

Hack, C. (2015). Analytical rubrics in higher education: A repository of empirical data. British Journal of Educational Technology, 46(5), 924-927. http://doi.org/10.1111/ bjet.12304

Ibarra-Sáiz, M.S., \& Rodríguez-Gómez, G. (2014a). Aportaciones para la formación del profesorado y estudiantes de Educación Superior en e-Evaluación: herramientas tecnológicas facilitadoras. En Proceedings of the VII International GUIDE Conference Cultura en la modernización global: El papel de la educación a distancia. Recuperado de https://goo.gl/btq09m

Ibarra-Sáiz, M.S., \& Rodríguez-Gómez, G. (2014b). Formación del profesorado universitario en evaluación: análisis y prospectiva del Programa Formativo EVAPESDevalSimWeb "Evaluación para el aprendizaje en la Educación Superior". En Congreso Iberoamericano de Ciencia, Tecnología, Innovación y Educación. Buenos Aires. Organización de Estados Iberoamericanos para la Educación, la Ciencia y la Cultura. Recuperado de https://goo.gl/MFmbEa_

Ibarra-Sáiz, M.S. \& Rodríguez-Gómez, G. (2017). EvalCOMIX®: a Web-Based Programme to Support Collaboration in Assessment. En T. Issa, P. Kommers, T. Issa, P. Isaías, \& T.B. Issa (Eds.), Smart Technology Applications in Business Environments (pp. 249-275). Hershey, PA: IGI Global.

Ibarra-Sáiz, M.S., Rodríguez-Gómez, G., \& García-Jiménez, E. (2013). DevalSimWeb_ Catalogue of transversal assessment skills. Cadiz: EVALfor Research Group.

Ibarra-Sáiz, M.S., Rodríguez-Gómez, G., \& García-Jiménez, E. (2016). Tareas de calidad: Caracterización I. [Microvídeo]. Cádiz: EVALfor - Grupo de Investigación. Recuperado de https://goo.gl/f5hP58

Joint Committee on Standards for Educational Evaluation. (2002). The student evaluation standard: How to improve evaluations of students. Thousands Oaks, CA: Corwin.

Jonsson, A., \& Svingby, G. (2007). The Use of Scoring Rubrics: Reliability, Validity and Educational Consequences. Educational Research Review, (2), 130-144. doi: http:// dx.dx.doi.org/10.1016/j.edurev.2007.05.002

Kamphorst, J., Hofman, W., Jansen, E., \& Terlouw, C. (2013). The relationship between perceived competence and earned credits in competence-based higher education. Assessment E Evaluation in Higher Education 38(6), 646-661. doi: http://dx.doi.org/1 0.1080/02602938.2012.680015

Lester, S. (2014). Professional competence standards and frameworks in the United Kingdom. Assessment \& Evaluation in Higher Education 39(1), 38-52. doi:10.1080/02 602938.2013.792106

Litwin, M. S. (2003). How to Assess and Interpret Survey Psychometrics. Thousands Oaks, CA: SAGE Publications.

Liu, X., \& Li, L. (2014). Assessment training effects on student assessment skills and task performance in a technology-facilitated peer assessment. Assessment E Evaluation in Higher Education, 39(3), 275-292. doi: http://dx.doi.org/10.1080/02602938.2013.823540 
Menéndez-Varela, J.L., \& Gregori-Giralt, E. (2016). The contribution of rubrics to the validity of performance assessment: a study of the conservation-restoration and design undergraduate degrees. Assessment $\mathcal{E}$ Evaluation in Higher Education, 41(2), 228-244. doi: http://doi.org/10.1080/02602938.2014.998169

Mueller, D., \& Strohmeier, S. (2011). Design characteristics of virtual learning environments: state of research. Computers $\mathcal{E}$ Education 57(4), 2505-2516. doi: http:// dx.doi.org/10.1016/j.compedu.2011.06.017

Olmos Migueláñez, S., \& Rodríguez Conde, M.J. (2011). Perspectiva tecnológica de la evaluación educativa en la universidad. Teoría de la Educación 23(1), 131-157. Recuperado de http://campus.usal.es/ revistas_trabajo/index.php/1130-3743/article/ viewFile/8581/8813

Peris Ortiz, M., \& Merigó Lindahl, J.M. (Eds.). (2015). Sustainable learning in higher education, innovation, technology, and knowledge management. London: Springer-Verlag. doi: http://dx.doi.org/10.1007/978-3-319-10804-9_1

Poblete, M., \& Villa, A. (2011). SEBSCO, una experiencia alternativa para evaluar competencias. Aula Abierta, 39(3), 15-30. Recuperado de https://dialnet.unirioja.es/ servlet/articulo?codigo $=3691465$

Redecker, C., \& Johannessen, O. (2013). Changing assessment-Towards a new assessment paradigm using ICT. European Journal of Education, 48(1), 79-96. doi: http://dx.doi.org/10.1111/ejed.12018

Reddy, Y., \& Andrade, H. (2010). A Review of Rubric Use in Higher Education. Assessment $\mathcal{E}$ Evaluation In Higher Education, 35(4), 435-448. doi: http://dx.doi. org/10.1080/02602930902862859

Rodríguez-Gómez, G., \& Ibarra-Sáiz, M.S. (Eds.) (2011). e-Evaluación orientada al e-Aprendizaje estratégico en la Universidad. Madrid: Narcea.

Rodríguez-Gómez, G., \& Ibarra-Sáiz, M.S. (2015). Assessment as Learning and Empowerment: Towards Sustainable Learning in Higher Education. En M. PerisOrtiz \& J. M. Merigó Lindahl (Eds.), Sustainable Learning in Higher Education. Developing Competencies for the Global Marketplace (pp. 1-20). Springer International Publishing. doi: http://dx.doi.org/10.1007/978-3-319-10804-9_1

Rodríguez-Gómez, G., Ibarra-Sáiz, M.S., \& Cubero-Ibáñez, J. (2018). Competencias básicas relacionadas con la evaluación. Un estudio sobre la percepción de los estudiantes universitarios. Educación XXI, 21(1), 181-208. doi: http://dx.doi.org/10.5944/ educXX1.14457

Rubio, M.J., Tocaín Garzón, A.L., \& Ruiz Quiranza, R. (2014). Programa formativo APREVAL DevalSimWeb: aprender evaluando en la Educación Superior: manual del formador. Ecuador: Ibarra.

Sliney, A., \& Murphy, D. (2011). Using serious games for assessment. In M. Ma, A. Oikonomou, \& L. C. Jain (Eds.), Serious Games and Edutainment Applications (pp. 225-243). Springer. doi: 10.1007/978-1-4471-2161-9_12

Skjong, R. \& Wentworth, B. (2000). Expert Judgement and Risk Perception. Recuperado de http://research.dnv.com/skj/Papers/SkjWen.pdf

Tejada, J., \& Ruiz, C. (2016). Evaluación de competencias profesionales en Educacion Superior: Retos e implicaciones. Educación XXI, 19(1), 17-38. doi: http://dx.doi. org/10.5944/educXX1.12175 
Tomas, C., Borg, M., \& McNeil, J. (2015). E-assessment: Institutional development strategies and the assessment life cycle. British Journal of Educational Technology, 46(3), 588-596. doi: http://dx.doi.org/10.1111/bjet.12153

Villa, A., \& Poblete, M. (2007). Aprendizaje Basado en Competencias. Una propuesta para la evaluación de las competencias genéricas. Bilbao: Mensajero.

Villa, A., \& Poblete, M. (2011). Evaluación de competencias genéricas: principios, oportunidades y limitaciones. Bordón: Revista de Pedagogía, 63(1), 147-170. Recuperado de http://www.innova.deusto.es/images/archivos/evaluaci\%C3\%B3n_competencias_ aurelio_bordon.pdf

Villardón, L. (2006). Evaluación del aprendizaje para promover el desarrollo de competencias. Educatio Siglo XXI, 24. Recuperado de http://revistas.um.es/educatio/ article/view/153

Zabala, A., \& Arnau, L. (2008). Evaluar competencias es evaluar procesos en la resolución de situaciones problema. En A. Zabala y L. Arnau, 11 Ideas clave: cómo aprender y enseñar competencias. Barcelona: Graó.

Zlatkin-Troitschanskaia, O., Shavelson, R.J., \& Kuhn, C. (2015). The international state of research on measurement of competency in higher education. Studies in Higher Education 40(3), 393-411. doi: http://dx.doi.org/10.1080/03075079.2015.1004241

\section{Agradecimientos}

Este trabajo ha sido posible gracias al Proyecto DevalSimWeb Desarrollo de competencias profesionales a través de la evaluación participativa y la simulación utilizando herramientas web. № de contrato DCI-ALA/19.09.01/11/21526/264-773/ALFAIII(2011)-10. Programa de cooperación entre la Unión Europea y América Latina en materia de Educación Superior, ALFA III (2007-2013). Comisión Europea, Dirección General de Desarrollo y Cooperación, EuropaAid América Latina y el Caribe. Socios: Universidad de CádizEspaña (Coordinadora), Durham University-United Kingdom, Pontifica Universidad Católica de Ecuador-Sede Ibarra, Universidad de Antioquia-Colombia, Universidad de Costa Rica y Universidad Nacional Agraria de Nicaragua.

Queremos mostrar nuestro agradecimiento a Eduardo García Jiménez y Laura Sánchez Calleja por su colaboración en el proceso de diseño de la propuesta, así como a los validadores externos del diseño.

Fecha de recepción: 21 de diciembre de 2016.

Fecha de revisión: 18 de abril de 2017.

Fecha de aceptación: 11 de mayo de 2017. 


\section{Apéndice: Descripción de tareas de evaluación y e-rúbricas}

Descripción de las tareas complejas de la competencia Juicio analítico y crítico

\begin{tabular}{|c|c|c|}
\hline \multicolumn{3}{|c|}{ Juicio analítico y crítico } \\
\hline & Evaluación inicial & Evaluación final \\
\hline Situación/problema & $\begin{array}{l}\text { Articulo "¿Las armas matan o } \\
\text { disuaden?" en el que su autor } \\
\text { muestra diferentes } \\
\text { argumentos a favor y en } \\
\text { contra acerca de la tenencia de } \\
\text { armas. }\end{array}$ & $\begin{array}{l}\text { Artículo "Ley, ciencia y conciencia } \\
\text { ante el aborto" en el que su autor } \\
\text { se apoya en aspectos legales, } \\
\text { científicos y morales para } \\
\text { argumentar su opinión sobre el } \\
\text { aborto. }\end{array}$ \\
\hline Recurso & $\begin{array}{l}\text { Articulo web de opinión } \\
\text { Esplugas, A. (1 de diciembre } \\
\text { de 2012). ¿Las armas matan o } \\
\text { disuaden? Libertad Digital. } \\
\text { Recuperado de } \\
\text { https://goo.gl/9gMiZ }\end{array}$ & $\begin{array}{l}\text { Artículo web de opinión } \\
\text { Masiá, J. (13 de mayo de 2013). } \\
\text { Ley, ciencia y conciencia ante el } \\
\text { aborto. El País. Recuperado de } \\
\text { https://goo.g1/nm0ft }\end{array}$ \\
\hline $\begin{array}{l}\text { Actividad de } \\
\text { evaluación }\end{array}$ & $\begin{array}{l}\text { Redacción de su opinión } \\
\text { respecto al tema que se } \\
\text { aborda en el mismo. }\end{array}$ & $\begin{array}{l}\text { Redacción de su opinión respecto } \\
\text { al tema que se aborda en el } \\
\text { mismo. }\end{array}$ \\
\hline
\end{tabular}

\begin{tabular}{|c|c|c|c|c|c|c|c|c|c|c|c|c|}
\hline \multicolumn{13}{|c|}{ Rúbrica para evaluar la competencia Juicio analitico y crítico } \\
\hline $100 \%$ & $\begin{array}{l}\text { Nivel } \\
\text { competencial } \\
\text { de Juicio } \\
\text { analitico y } \\
\text { critico }\end{array}$ & \multicolumn{5}{|c|}{ Cotidimo } & \multicolumn{3}{|c|}{ Rechesivo } & \multicolumn{3}{|c|}{ Congrensivo } \\
\hline \multicolumn{13}{|l|}{$33 \%$} \\
\hline $100 \%$ & Cuestionamiento & \multicolumn{5}{|c|}{$\begin{array}{l}\text { - Realiza preguntas relevantes. - Tiene en } \\
\text { cuenta el reconocimiento de las } \\
\text { condiciones del contexto para evitar } \\
\text { generalizar. }\end{array}$} & \multicolumn{3}{|c|}{$\begin{array}{l}\text { - Se plantea su propia perspectiva. - Explora distintas } \\
\text { creencias, argumentos y teorias para desarrollar su } \\
\text { propia perspectiva. }\end{array}$} & \multicolumn{3}{|c|}{$\begin{array}{l}\text {-Se cuestiona la situación dada. Plantea y argumenta } \\
\text { posibilidades y alternativas que abarquen a las distintas } \\
\text { audiencias implicadas en dicha situación. }\end{array}$} \\
\hline & & $\begin{array}{l}0 \\
0\end{array}$ & $\begin{array}{c}10 \\
0 \\
\end{array}$ & 20 & $\begin{array}{r}30 \\
0\end{array}$ & $\begin{array}{c}40 \\
0 \\
\end{array}$ & $\begin{array}{r}50 \\
0 \\
\end{array}$ & $\begin{array}{c}60 \\
0 \\
\end{array}$ & $\begin{array}{r}70 \\
0 \\
\end{array}$ & $\begin{array}{l}80 \\
0 \\
\end{array}$ & $\begin{array}{r}90 \\
0\end{array}$ & $\begin{array}{c}100 \\
0\end{array}$ \\
\hline \multicolumn{13}{|l|}{$33 \%$} \\
\hline $100 \%$ & Argumentación & \multicolumn{5}{|c|}{$\begin{array}{l}\text { - Acepta y reconoce el valor de las } \\
\text { criticas. - Explora anternativas y } \\
\text { posibilidades de análisis para defender } \\
\text { sus puntos de vista. }\end{array}$} & \multicolumn{3}{|c|}{$\begin{array}{l}\text { - Sigue una lógica argumentativa para defender su } \\
\text { razonamiento. - Valora perspectivas, interpretaciones y } \\
\text { teorias diversas para construir la logica de su } \\
\text { argumento. }\end{array}$} & \multicolumn{3}{|c|}{$\begin{array}{l}\text { - Muestra y utiliza habilidades de argumentación. - Es } \\
\text { capaz de clarificar conceptos, hacer distinciones y } \\
\text { establecer conexiones para fundamentar sus } \\
\text { conclusiones. }\end{array}$} \\
\hline & & $\begin{array}{l}0 \\
0 \\
\end{array}$ & $\begin{array}{c}10 \\
\end{array}$ & $\begin{array}{r}20 \\
0 \\
\end{array}$ & $\begin{array}{r}30 \\
0 \\
\end{array}$ & $\begin{array}{c}40 \\
\end{array}$ & $\begin{array}{l}50 \\
0 \\
\end{array}$ & $\begin{array}{c}60 \\
0 \\
\end{array}$ & $\begin{array}{r}70 \\
0 \\
\end{array}$ & $\begin{array}{r}80 \\
0 \\
\end{array}$ & $\begin{array}{l}90 \\
0 \\
\end{array}$ & $\begin{array}{c}100 \\
0\end{array}$ \\
\hline \multicolumn{13}{|l|}{$33 \%$} \\
\hline $100 \%$ & Crnicismo & \multicolumn{5}{|c|}{$\begin{array}{l}\text { - Se cuestiona lo que sucede. - Trata de } \\
\text { averiguar cual es el fundamento racional } \\
\text { de lo que se conoce o de nuestros actos. }\end{array}$} & \multicolumn{3}{|c|}{$\begin{array}{l}\text { - Desarrolla estrategias cognitivas y metodológicas } \\
\text { para cuestionar lo que sucede. - Profundiza en las } \\
\text { razones y argumentos que justifican lo que sucede. }\end{array}$} & \multicolumn{3}{|c|}{$\begin{array}{l}\text { - Se cuestiona su propia forma de ver lo que sucede.- } \\
\text { Elabora juicios propios que le permitan encontrar } \\
\text { soluciones alternativas que le ayuden a mejorar sus } \\
\text { acciones. }\end{array}$} \\
\hline & & $\begin{array}{l}0 \\
0\end{array}$ & $\begin{array}{c}10 \\
0\end{array}$ & 20 & $\begin{array}{r}30 \\
0\end{array}$ & 40 & $\begin{array}{c}50 \\
0\end{array}$ & 60 & $\begin{array}{r}70 \\
0\end{array}$ & 80 & 90 & 100 \\
\hline
\end{tabular}


Descripción de las tareas complejas de la competencia Sentido ético

\begin{tabular}{|c|c|c|}
\hline \multicolumn{3}{|c|}{ Sentido ético } \\
\hline & Evaluación inicial & Evaluación final \\
\hline Situación/problema & $\begin{array}{l}\text { Un farmaceútico descubre el } \\
\text { medicamento que puede } \\
\text { salvar la vida de una mujer } \\
\text { enferma. La medicina es cara } \\
\text { porque el farmaceútico está } \\
\text { cobrando diez veces lo que le } \\
\text { costó hacerla. El marido de la } \\
\text { mujer enferma, solo ha podido } \\
\text { reunir la mitad de lo que } \\
\text { cuesta. Le dice al farmaceútico } \\
\text { que su mujer se está muriendo } \\
\text { y le pide que le venda el } \\
\text { medicamento más barato o le } \\
\text { deje pagar más tarde. El } \\
\text { farmacéutico se niega y, ante } \\
\text { esto, el marido desesperado } \\
\text { piensa atracar la farmacia para } \\
\text { robar la medicina. }\end{array}$ & $\begin{array}{l}\text { Luis cuando tenía } 18 \text { años estaba } \\
\text { metido en la droga y en compañía } \\
\text { de otros jóvenes asaltó la vivienda } \\
\text { de una mujer, viuda y madre de } \\
\text { dos niños pequeños, y le robaron } \\
2000 \text { euros que la mujer tenía para } \\
\text { pagar el colegio de los sus hijos, } \\
\text { además de algunos objetos de } \\
\text { valor y recuerdos familiares. En } \\
2007 \text { fue condenado a más de dos } \\
\text { años de prisión. La sentencia fue } \\
\text { recurrida y el supremo ratificó la } \\
\text { condena } 7 \text { años después. Luis en } \\
\text { este tiempo se ha casado, tiene un } \\
\text { hijo y trabajo. Su abogado ha } \\
\text { pedido el indulto alegando que ya } \\
\text { está reinsertado en la sociedad. }\end{array}$ \\
\hline Recurso & $\begin{array}{l}\text { Texto escrito (Adquisición } \\
\text { medicamento) }\end{array}$ & Texto escrito (sentencia judicial) \\
\hline $\begin{array}{l}\text { Actividad de } \\
\text { evaluación }\end{array}$ & $\begin{array}{l}\text { ¿Qué haría en lugar del } \\
\text { marido? Justifique su } \\
\text { respuesta. } \\
\text {-Redacte su opinión respecto } \\
\text { al dilema planteado. }\end{array}$ & $\begin{array}{l}\text { - ¿Se le debe indultar? Justifique su } \\
\text { respuesta. } \\
\text {-Redacte su opinión respecto al } \\
\text { dilema planteado. }\end{array}$ \\
\hline
\end{tabular}

\begin{tabular}{|c|c|c|c|c|c|c|c|c|c|c|c|c|}
\hline \multicolumn{13}{|c|}{ Rúbrica para evaluar la competencia Sentido ético } \\
\hline $100 \%$ & \begin{tabular}{|l|} 
Nivel \\
competencial \\
de Sentido \\
ético
\end{tabular} & \multicolumn{5}{|c|}{ Cotidiano } & & Reflexh & & \multicolumn{3}{|c|}{ Comprensivo } \\
\hline \multicolumn{13}{|l|}{$33 \%$} \\
\hline $100 \%$ & $\begin{array}{l}\text { Conflicto de } \\
\text { intereses }\end{array}$ & \multicolumn{5}{|c|}{$\begin{array}{l}\text { Reconoce conflictos de conciencia y } \\
\text { tiene la habilidad para encontrar } \\
\text { posibles respuestas a los mismos. }\end{array}$} & \multicolumn{3}{|c|}{$\begin{array}{l}\text { Ejercita el control de posibles confictos de } \\
\text { intereses en diferentes situaciones. }\end{array}$} & \multicolumn{3}{|c|}{$\begin{array}{l}\text { Valora las situaciones sociales que presentan dificultades } \\
\text { morales desde diferentes perspectivas, y considera otras } \\
\text { logicas y niveles de conocimiento. }\end{array}$} \\
\hline & & 0 & 10 & 20 & 30 & 40 & 50 & 60 & $\begin{array}{c}70 \\
0\end{array}$ & 80 & 90 & 100 \\
\hline \multicolumn{13}{|l|}{$33 \%$} \\
\hline $100 \%$ & Credibilidad & \multicolumn{5}{|c|}{$\begin{array}{l}\text { Antes de hacer algo, considera si } \\
\text { posee todo lo que necesita para actuar } \\
\text { responsablemente. }\end{array}$} & \multicolumn{3}{|c|}{$\begin{array}{l}\text { Antes de actuar, recaba evidenclas que le } \\
\text { permitan responder correctamente ante la } \\
\text { situación a la que se enfrenta. }\end{array}$} & \multicolumn{3}{|c|}{$\begin{array}{l}\text { Considera otros puntos de vista, entendiendo los argumentos o } \\
\text { explicaciones que hay detras de cada postura o juicio, y realiza } \\
\text { revisiones criticas de cada una de ellos. }\end{array}$} \\
\hline & & 0 & 10 & 20 & 30 & 40 & 50 & 60 & 70 & 80 & 90 & 100 \\
\hline \multicolumn{13}{|l|}{$33 \%$} \\
\hline $100 \%$ & Sesgo & \multicolumn{5}{|c|}{$\begin{array}{l}\text { Reconoce que pueden existir factores } \\
\text { arbitrarios que influyen on sus } \\
\text { valoraciones. }\end{array}$} & \multicolumn{3}{|c|}{$\begin{array}{l}\text { Utiliza criterios defendibles e información } \\
\text { valida para evitar la influencia de factores } \\
\text { arbitrarios en las valoraciones. }\end{array}$} & \multicolumn{3}{|c|}{$\begin{array}{l}\text { Incorpora e integra la existencia de valores y practicas de otros } \\
\text { contextos aportandoles otros sistemas eticos. }\end{array}$} \\
\hline & & 0 & 10 & 20 & 30 & 40 & 50 & 60 & $\begin{array}{r}70 \\
0\end{array}$ & 80 & 90 & 100 \\
\hline
\end{tabular}


Descripción de las tareas complejas de la competencia Resolución de problemas

\begin{tabular}{|c|c|c|}
\hline \multicolumn{3}{|c|}{ Resolución de problemas } \\
\hline & Evaluación inicial & Evaluación final \\
\hline Situación/problema & $\begin{array}{l}\text { En esta tarea partimos de su } \\
\text { propia experiencia. Seleccione } \\
\text { una situación-problema que } \\
\text { haya vivenciado en el pasado, } \\
\text { y a partir de la misma vaya } \\
\text { respondiendo a la actividad de } \\
\text { evaluación propuesta. }\end{array}$ & $\begin{array}{l}\text { Tenemos cinco casas de cinco } \\
\text { colores diferentes y en cada una de } \\
\text { ellas vive una persona de una } \\
\text { nacionalidad diferente. } \\
\text { Cada uno de los dueños bebe una } \\
\text { bebida diferente, fuma una marca } \\
\text { de cigarrillos diferente y tiene una } \\
\text { mascota diferente. Después de } \\
\text { aportar diferentes claves, la } \\
\text { pregunta es: ¿De quién es el } \\
\text { pececito? }\end{array}$ \\
\hline Recurso & $\begin{array}{l}\text { Texto escrito (presentación } \\
\text { qué hiciste) }\end{array}$ & $\begin{array}{l}\text { Texto escrito (El acertijo de } \\
\text { Einstein) Recuperado de } \\
\text { https://goo.gl/f01xVP }\end{array}$ \\
\hline $\begin{array}{l}\text { Actividad de } \\
\text { evaluación }\end{array}$ & $\begin{array}{l}\text {-Describa la situación- } \\
\text { problema } \\
\text { ¿Qué pasos siguió para } \\
\text { solventarlo? } \\
-¿ \text { Ha aplicado alguna vez este } \\
\text { mismo procedimiento en la } \\
\text { resolución de otro problema? } \\
\text { Explíquelo. } \\
\text {-¿En qué otra situación cree } \\
\text { que podría aplicar este mismo } \\
\text { método? }\end{array}$ & $\begin{array}{l}\text {-Intente resolver el problema y } \\
\text { explique qué pasos ha dado para } \\
\text { lograrlo: } \\
\text { ¿Cuál es el problema? } \\
\text { ¿Qué pasos ha seguido para } \\
\text { solventarlo? (Explique } \\
\text { detalladamente cada uno de ellos) } \\
\text { ¿Ha aplicado alguna vez este } \\
\text { mismo procedimiento en la } \\
\text { resolución de otro problema? } \\
\text { Explíquelo. } \\
\text { ¿En qué otra situación cree que } \\
\text { podría aplicar este mismo } \\
\text { método? }\end{array}$ \\
\hline
\end{tabular}

\begin{tabular}{|c|c|c|c|c|c|c|c|c|c|c|c|c|}
\hline \multicolumn{13}{|c|}{ Rubrica para evaluar la competencia Resolución de problemas } \\
\hline $100 \%$ & $\begin{array}{l}\text { Nivel } \\
\text { competencial } \\
\text { Resolución } \\
\text { de } \\
\text { problemas }\end{array}$ & \multicolumn{5}{|c|}{ Cotidiano } & \multicolumn{3}{|c|}{ Reflexivo } & \multicolumn{3}{|c|}{ Comprensivo } \\
\hline \multicolumn{13}{|l|}{$33 \%$} \\
\hline \multirow[t]{2}{*}{1005} & $\begin{array}{l}\text { Comprension } \\
\text { del problema }\end{array}$ & \multicolumn{5}{|c|}{$\begin{array}{l}\text { Reconoce los rasgos distintivos de un problema. } \\
\text { aunque no conoce muy blen cómo ha llegado a } \\
\text { identficario, intulitvamente sabe que en esa } \\
\text { situacion o explicacion hay algo que no es } \\
\text { coherente. }\end{array}$} & \multicolumn{3}{|c|}{$\begin{array}{l}\text { Anallza de forma sistematica el problema } \\
\text { tomando en consideración que pregunta } \\
\text { hay que resolver, que variables o } \\
\text { dimensiones tieno el problema. como debe } \\
\text { ser la respuesta. etc. }\end{array}$} & \multicolumn{3}{|c|}{$\begin{array}{l}\text { Reconoce las caracteristicas del problema y busca problemas } \\
\text { simllares que le sirvan de referencia. }\end{array}$} \\
\hline & & 0 & 10 & 20 & 30 & 40 & 50 & 60 & 70 & 80 & 90 & 100 \\
\hline \multicolumn{13}{|l|}{$33 \%$} \\
\hline $100 \%$ & $\begin{array}{l}\text { Planificacion y } \\
\text { ejecucion }\end{array}$ & \multicolumn{5}{|c|}{$\begin{array}{l}\text { Establece un plan de acción para resolver el } \\
\text { problema apoyandose en las soluciones } \\
\text { aportadas por otros que le resultan adecuadas. } \\
\text { Hay un modelo experiencial, pero no teórico de } \\
\text { resolución de problemas. Uleva a cabo el plan de } \\
\text { accion disefiado }\end{array}$} & \multicolumn{3}{|c|}{$\begin{array}{l}\text { Define o elige una estrategia para } \\
\text { enfrentarse al problema tomando como } \\
\text { punto de partida un referente teorico que } \\
\text { indica que pasos deben darse para } \\
\text { solucionar el problema. Lleva a cabo la } \\
\text { estrategia escogida. }\end{array}$} & \multicolumn{3}{|c|}{$\begin{array}{l}\text { Examina diterentes posibilidades para construir o elegir un modo } \\
\text { de resolucion poniendo a prueba distintas estrategias y } \\
\text { comparandolas entre si para ver cual es la mas acertada. } \\
\text { Resuelve el problema }\end{array}$} \\
\hline & & 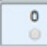 & 10 & 20 & 30 & 40 & 50 & 60 & 70 & 80 & 90 & 100 \\
\hline \multicolumn{13}{|l|}{$33 \%$} \\
\hline \multirow[t]{2}{*}{$100 \%$} & $\begin{array}{l}\text { Revision y } \\
\text { transterencia }\end{array}$ & \multicolumn{5}{|c|}{$\begin{array}{l}\text { Analiza la solucion dada al problema y se } \\
\text { pregunta en que medida puede aplicarlo a } \\
\text { situaciones similares. }\end{array}$} & \multicolumn{3}{|c|}{$\begin{array}{l}\text { Cuestiona la adecuacion de la respuesta } \\
\text { que ha dado para resolver el problema } \\
\text { preguntandose si hay una manera mas } \\
\text { sencilla de solucionarlo. Y se plantea la } \\
\text { posibilidad de aplicar esta resolucion en } \\
\text { situaciones similares. }\end{array}$} & \multicolumn{3}{|c|}{$\begin{array}{l}\text { Analiza alternativas a la respuesta dada al problema (teniendo } \\
\text { en cuenta que tipos de conocimiento y que consecuencias } \\
\text { sociales estan asociados a cada una de estas alternativas) y } \\
\text { establece un procedimiento para determinar cuando la } \\
\text { alternativa adoptada para un contexto determinado, es } \\
\text { transferible a otro contexto. }\end{array}$} \\
\hline & & 8 & 10 & 20 & 30 & 40 & 50 & 60 & 70 & 80 & 90 & 100 \\
\hline
\end{tabular}


Descripción de las tareas complejas de la competencia Toma de decisiones

\begin{tabular}{|c|c|c|}
\hline \multicolumn{3}{|c|}{ Toma de decisiones } \\
\hline & Evaluación inicial & Evaluación final \\
\hline Situación/problema & $\begin{array}{l}\text { E1 } 4 \text { de noviembre de } 1979 \text {, } \\
\text { cuando la revolución iraní } \\
\text { alcanza su momento de mayor } \\
\text { tensión, unos militantes } \\
\text { irrumpen en la embajada } \\
\text { norteamericana en Teherán, } \\
\text { tomando a } 52 \text { norteamericanos } \\
\text { como rehenes. Pero en medio } \\
\text { del caos, seis de ellos } \\
\text { consiguen escabullirse y } \\
\text { encuentran refugio en la } \\
\text { embajada canadiense. Para } \\
\text { salir del país con vida, deben } \\
\text { decidir qué hacer. }\end{array}$ & $\begin{array}{l}\text { Tres amigos caminando por el } \\
\text { bosque encuentran de casualidad, } \\
\text { una avioneta accidentada bajo la } \\
\text { nieve. Su piloto ha fallecido, pero } \\
\text { en el aeroplano hallan una maleta } \\
\text { llena de dinero. A continuación } \\
\text { vamos a observar un video en el } \\
\text { que se muestra esta secuencia y lo } \\
\text { que sucede a partir de este } \\
\text { momento. }\end{array}$ \\
\hline Recurso & $\begin{array}{l}\text { Fragmento película. } \\
\text { Affleck, B. (escritor } \\
\text { y director). (2012). Argo. USA: } \\
\text { Warner Bros. }\end{array}$ & $\begin{array}{l}\text { Fragmento pelicula. } \\
\text { Raimi, S. (director). (1998). A simple } \\
\text { plan.USA: Paramount Pictures. }\end{array}$ \\
\hline $\begin{array}{l}\text { Actividad de } \\
\text { evaluación }\end{array}$ & $\begin{array}{l}\text {-Enumere las actuaciones de } \\
\text { los protagonistas de la película } \\
\text { que, en su opinión, son } \\
\text { necesarias para una buena } \\
\text { toma decisiones. } \\
\text {-Describa aquellas actuaciones } \\
\text { que crea necesarias para una } \\
\text { buena toma de decisiones que } \\
\text { no aparecen reflejadas en el } \\
\text { vídeo. }\end{array}$ & $\begin{array}{l}\text {-Enumere y describa qué } \\
\text { actuaciones llevaría a cabo para } \\
\text { tomar la decisión antes indicada. }\end{array}$ \\
\hline
\end{tabular}

\begin{tabular}{|c|c|c|c|c|c|c|c|c|c|c|c|c|}
\hline \multicolumn{13}{|c|}{ Rúbrica para evaluar la competencia Toma de decisiones } \\
\hline $100 \%$ & \begin{tabular}{l|} 
Nivel \\
competencial \\
de Toma de \\
decisiones
\end{tabular} & \multicolumn{5}{|c|}{ Cotidieno } & \multicolumn{3}{|c|}{ Rentexvo } & \multicolumn{3}{|c|}{ Comprensivo } \\
\hline \multicolumn{13}{|l|}{$33 \%$} \\
\hline \multirow[t]{2}{*}{$100 \%$} & \multirow[t]{2}{*}{$\begin{array}{l}\text { Consistencia } \\
\text { en las } \\
\text { valoraciones }\end{array}$} & \multicolumn{5}{|c|}{$\begin{array}{l}\text { - Antes de tomar la decisión se cuestiona ia } \\
\text { opción, repasando cumo ha llegado a la } \\
\text { misma. -Tiene en cuenta las diferentes } \\
\text { opciones que le ofrecen las personas de su } \\
\text { entorno. }\end{array}$} & \multicolumn{3}{|c|}{$\begin{array}{l}\text { - Expone y defende la opción tomada de forma convincente y } \\
\text { constructiva, revisando la lógica que ha seguido. - Tiene en } \\
\text { cuenta las distintas apreciaciones que realizan otras personas y } \\
\text { las contrasta para asegurarse que responde a la logica seguida. }\end{array}$} & \multicolumn{3}{|c|}{$\begin{array}{l}\text { - Somete a revision la logica predominante } \\
\text { en su contexto para valorar diferentes } \\
\text { aspectos que pueden infuir. - Propone y } \\
\text { debate nuevas formas o alternativas. }\end{array}$} \\
\hline & & $\begin{array}{l}0 \\
0\end{array}$ & $\begin{array}{c}10 \\
0\end{array}$ & $\begin{array}{c}20 \\
0\end{array}$ & $\begin{array}{r}30 \\
0\end{array}$ & 40 & $\begin{array}{l}50 \\
0\end{array}$ & 60 & $\begin{array}{c}70 \\
0\end{array}$ & $\begin{array}{c}80 \\
0\end{array}$ & $\begin{array}{c}90 \\
0\end{array}$ & $\begin{array}{c}100 \\
0\end{array}$ \\
\hline \multicolumn{13}{|l|}{$33 \%$} \\
\hline \multirow[t]{2}{*}{$100 \%$} & \multirow[t]{2}{*}{$\begin{array}{l}\text { Información } \\
\text { relevante y } \\
\text { conflable }\end{array}$} & \multicolumn{5}{|c|}{$\begin{array}{l}\text { Adopta una actitud escéptica ante las } \\
\text { informaciones que recibe y sopesa su } \\
\text { relevancia para justificar la opción elegida. }\end{array}$} & \multicolumn{3}{|c|}{$\begin{array}{l}\text { Comprueba si la información recogida satisface las necesidades } \\
\text { de la situación a la que tiene que responder y revisa si es válida y } \\
\text { fiable. }\end{array}$} & \multicolumn{3}{|c|}{$\begin{array}{l}\text { Se cuestiona la relevancia de la } \\
\text { información integrando fuentes diversas } \\
\text { para determinar qué intormación es } \\
\text { realmente importante, contrastándolas e } \\
\text { integrándolas. }\end{array}$} \\
\hline & & $\begin{array}{l}0 \\
0\end{array}$ & 10 & 20 & $\begin{array}{c}30 \\
0\end{array}$ & 40 & 50 & $\begin{array}{c}60 \\
0\end{array}$ & $\begin{array}{c}70 \\
0\end{array}$ & 80 & 90 & 100 \\
\hline \multicolumn{13}{|l|}{$33 \%$} \\
\hline \multirow[t]{2}{*}{$100 \%$} & $\begin{array}{l}\text { Resultados o } \\
\text { informes de } \\
\text { evaluaciones } \\
\text { eficaces }\end{array}$ & \multicolumn{5}{|c|}{$\begin{array}{l}\text { Se cuestiona cuál es la utilidad o valor } \\
\text { práctico de la decisión tomada para si mimo y } \\
\text { para los otros. }\end{array}$} & \multicolumn{3}{|c|}{$\begin{array}{l}\text { Analiza la relevancia de la información que se deriva de esa } \\
\text { stuación en función de las consecuencias que tendrá ahora o en } \\
\text { el futuro }\end{array}$} & \multicolumn{3}{|c|}{$\begin{array}{l}\text { Anticipa las consecuencias de las } \\
\text { decisiones asumiendo y compartiendo los } \\
\text { riesgos responsablemente. }\end{array}$} \\
\hline & & $\begin{array}{l}0 \\
0\end{array}$ & 10 & 20 & $\begin{array}{r}30 \\
0\end{array}$ & 40 & 50 & 60 & $\begin{array}{c}70 \\
0\end{array}$ & $\begin{array}{c}80 \\
0\end{array}$ & $\begin{array}{c}90 \\
0\end{array}$ & 100 \\
\hline
\end{tabular}


Descripción de las tareas complejas de la competencia Trabajo en equipo

\begin{tabular}{|c|c|c|}
\hline \multicolumn{3}{|c|}{ Trabajo en equipo } \\
\hline & Evaluación inicial & Evaluación final \\
\hline Situación/problema & $\begin{array}{l}\text { Un grupo de personas decide } \\
\text { montar un restaurante } \\
\text { temático. Para ello, forman un } \\
\text { equipo de trabajo. A } \\
\text { continuación vamos a } \\
\text { observar un video en el que se } \\
\text { muestra lo que ocurre en el } \\
\text { equipo a partir de ese } \\
\text { momento. }\end{array}$ & $\begin{array}{l}\text { Un grupo de personas decide } \\
\text { organizar actividades para turistas. } \\
\text { Para ello, forman un equipo de } \\
\text { trabajo. A continuacion vamos a } \\
\text { observar un video en el que se } \\
\text { muestra lo ocurre en el equipo } \\
\text { desde la primera toma de contacto } \\
\text { de los miembros del equipo. }\end{array}$ \\
\hline Recurso & $\begin{array}{l}\text { Fragmento programa de } \\
\text { television Basat, L. (director). } \\
\text { (2009). Semana 2. El Aprendiz. } \\
\text { La Sexta. Esparia. }\end{array}$ & $\begin{array}{l}\text { Fragmento programa de televisión } \\
\text { Basat, L. (director). } \\
\text { (2009). Semana 3. El Aprendiz. La } \\
\text { Sexta. España. }\end{array}$ \\
\hline $\begin{array}{l}\text { Actividad de } \\
\text { evaluación }\end{array}$ & $\begin{array}{l}\text { Identifique y enumere las } \\
\text { actuaciones de los } \\
\text { protagonistas del video que, } \\
\text { en su opinión, están } \\
\text { contribuyendo a que la tarea } \\
\text { que deben realizar (montar } \\
\text { restaurante temático) se } \\
\text { convierta en un verdadero } \\
\text { trabajo de equipo. } \\
\text {-Identifique y enumere las } \\
\text { actuaciones de los } \\
\text { protagonistas del video que, } \\
\text { en su } \\
\text { opinión, no están } \\
\text { contribuyendo a que dicha } \\
\text { tarea sea un trabajo en equipo }\end{array}$ & $\begin{array}{l}\text { Identifique y enumere las } \\
\text { actuaciones de los protagoristas } \\
\text { del video que, en su opinión, están } \\
\text { contribuyendo a que la tarea que } \\
\text { deben realizar (organizar } \\
\text { actividades para turistas) se } \\
\text { convierta en un verdadero trabajo } \\
\text { de equipo. } \\
\text {-Identifique y enumere las } \\
\text { actuaciones de los protagonistas } \\
\text { del video que, en su } \\
\text { opinión, no están contribuyendo a } \\
\text { que dicha tarea sea un trabajo en } \\
\text { equipo }\end{array}$ \\
\hline
\end{tabular}

